\title{
Caulobacter Lon protease has a critical role in cell-cycle control of DNA methylation
}

\author{
Rachel Wright, Craig Stephens, Gary Zweiger, ${ }^{1}$ Lucy Shapiro, ${ }^{3}$ and M.R.K. Alley ${ }^{2}$ \\ Department of Developmental Biology, Beckman Center for Molecular and Genetic Medicine, Stanford University, Stanford, \\ California 94305-5427 USA
}

CcrM, an adenine DNA methyltransferase, is essential for viability in Caulobacter crescentus. The CcrM protein is present only in the predivisional stage of the cell cycle, resulting in cell-cycle-dependent variation of the DNA methylation state of the chromosome. The availability of CcrM is controlled in two ways: (1) the ccrM gene is transcribed only in the predivisional cell, and (2) the CcrM protein is rapidly degraded prior to cell division. We demonstrate here that CcrM is an important target of the Lon protease pathway in $C$. crescentus. In a lon null mutant, ccrM transcription is still temporally regulated, but the CcrM protein is present throughout the cell cycle because of a dramatic increase in its stability that results in a fully methylated chromosome throughout the cell cycle. Because the Lon protease is present throughout the cell cycle, it is likely that the level of CcrM in the cell is controlled by a dynamic balance between temporally varied transcription and constitutive degradation. We have shown previously that restriction of CcrM to the C. crescentus predivisional cell is essential for normal morphogenesis and progression through the cell cycle. Comparison of the lon null mutant strain with a strain whose DNA remains fully methylated as a result of constitutive expression of $c c r M$ suggests that the effect of Lon on DNA methylation contributes to several developmental defects observed in the lon mutant. These defects include a frequent failure to complete cell division and loss of precise cell-cycle control of initiation of DNA replication. Other developmental abnormalities exhibited by the lon null mutant, such as the formation of abnormally long stalks, appear to be unrelated to altered chromosome methylation state. The Lon protease thus exhibits pleiotropic effects in $C$. crescentus growth and development.

[Key Words: C. crescentus; CcrM; DNA methyltransferase; Lon protease; cell cycle]

Received March 4, 1996; revised version accepted May 6, 1996.

Cells modulate regulatory circuits in response to environmental signals and programmed developmental cues. Regulatory circuits frequently involve changing the availability of specific proteins through the control of transcription and translation. Protein degradation, however, is emerging as an important regulatory component in many cellular processes. For example, several key regulators of the eukaryotic cell cycle, the activating and inhibiting partners of the cyclin-dependent kinases, are controlled by modulating their stability via the ubiquitin-mediated protein degradation pathway (for review, see King et al. 1994). In the bacterium Caulobacter crescentus, cell-cycle-dependent proteolysis of chemoreceptors and the flagellar motor protein FliF has been shown

Present addresses: 'Genentech, Inc., South San Francisco, California 94080-4990 USA; ${ }^{2}$ Department of Biochemistry, Imperial College, London, UK.

${ }^{3}$ Corresponding author. to be dependent on a proteolytic system that recognizes a short carboxy-terminal domain (Alley et al. 1992; Jenal and Shapiro 1996). We have recently reported that the essential, cell-cycle-regulated CcrM DNA methyltransferase in $C$. crescentus is quite unstable, suggesting that rapid and specific proteolysis may be a key element in the cell-cycle control of DNA methylation in this organism (Stephens et al. 1996). We describe here the identification of a protease, Lon, which is necessary for degradation of CcrM, and describe phenotypic properties of a lon null mutant demonstrating that Lon plays critical roles in C. crescentus growth and development.

The $C$. crescentus cell cycle (Fig. 1) involves a series of differentiation events culminating in the formation of two distinct progeny cells: a sessile stalked cell and a motile swarmer cell (for review, see Brun et al. 1994). The progeny swarmer cell contains a single polar flagellum and polar pili and is unable to initiate chromosomal DNA replication. In response to an unknown signal, swarmer cells differentiate into nonmotile stalked cells. During this transition the flagellum is shed, pili are lost, 


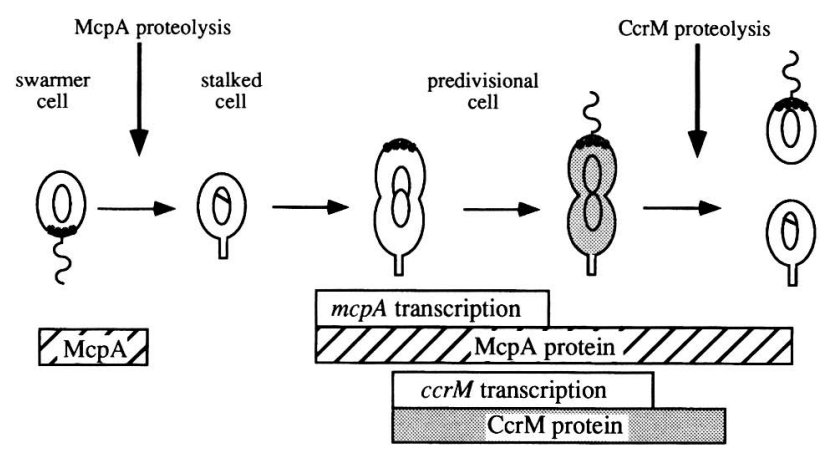

Figure 1. Diagram of the $C$. crescentus cell cycle indicating temporally controlled proteolysis of the McpA chemoreceptor and the CcrM DNA methyltransferase. A schematic diagram of the $C$. crescentus cell cycle is shown with the different cell types labeled. The solid areas at the pole of the swarmer and predivisional cells indicate the McpA chemoreceptor. The shading in the late predivisional cell indicates the presence of the CcrM protein during the cell cycle. The oval and $\theta$ figures within the cells represent the chromosome before and during replication, respectively. The open bars below the diagram represent the approximate time of $m c p A$ and $c c r M$ transcription, and hatched bars show the resulting McpA accumulation; the stippled bar shows CcrM protein accumulation. McpA and CcrM degradation during the cell cycle is indicated by arrows.

synthesis of a stalk occurs at the pole previously occupied by the flagellum, and chromosomal replication is initiated. As the stalked cell elongates, chemoreceptors, a new flagellum, and polar pili are synthesized at the pole opposite the stalk, yielding two distinct cell types upon division. These various differentiation events that occur during the $C$. crescentus cell cycle are associated with changes in gene expression and intracellular protein composition, resulting from both new protein synthesis and targeted protein degradation.

Replication of the chromosome in the stalked cell yields two hemimethylated daughter chromosomes, that is, chromosomes that are methylated only on the old DNA strand. Both daughter chromosomes become fully methylated just prior to cell division (Zweiger et al. 1994). The C. crescentus DNA methyltransferase CcrM catalyzes $\mathrm{N}^{6}$ methylation of the adenine residue in the sequence GAnTC (Zweiger et al. 1994). As with the Escherichia coli Dam DNA methyltransferase, CcrM does not have a cognate restriction enzyme (Zweiger et al. 1994; Stephens et al. 1996). The confinement of DNA methylation in C. crescentus to the late predivisional cell is controlled by regulation of the availability of the CcrM enzyme at the level of both transcription and protein stability (Fig. 1). Transcription of the $c c r M$ gene occurs only in the predivisional cell and is dependent on the global response regulator protein CtrA (Stephens et al. 1995; Quon et al. 1996). The CcrM protein is essentially restricted to the predivisional cell as a result of rapid proteolysis, with negligible levels detected in progeny cells after division (Fig. 1; Stephens et al. 1996). Cell- cycle control of DNA methylation in C. crescentus is required for normal progression through the cell cycle and proper cell division (Zweiger et al. 1994). If $c c r M$ is transcribed from the constitutively active $E$. coli lac promoter, yielding chromosomes that are fully methylated throughout the cell cycle, cells have an abnormal morphology: Division is often incomplete, cells tend to be twisted, and there is a relaxation in the control of the initiation of DNA replication (Zweiger et al. 1994). Thus, the normal progression through the cell cycle is in part dependent on ensuring that the CcrM DNA methyltransferase is present only during the predivisional stage. Identification of the protease responsible for removal of CcrM is an important step toward understanding control of these cell cycle events.

One of the most phylogenetically widespread proteases identified to date is the ATP-dependent serine protease Lon, which has been found in humans, yeast, and bacteria (for recent reviews, see Gottesman and Maurizi 1992; Goldberg et al. 1994). Primary sequences of Lon homologs are strongly conserved, particularly around the putative ATP-binding domain and the active site serine (Goldberg et al. 1994). The E. coli Lon protease is involved in the degradation of abnormal proteins (Gottesman and Maurizi 1992), and specific short-lived proteins such as SulA, RcsA, $\lambda \mathrm{N}$ and CcdA whose physiological function is dependent on their instability /Gottesman et al. 1981; Mizusawa and Gottesman 1983; Torres-Cabassa and Gottesman 1987; Van Melderen et al. 1994). Although the E. coli Lon protease is not normally required for growth, it is essential under certain conditions, such as for recovery after induction of the SOS response to DNA damage (Huisman et al. 1984; Schoemaker et al. 1984; Gottesman and Maurizi 1992). In Myxococcus xanthus, two lon genes have been isolated: lon $V$ is essential for vegetative growth (Tojo et al. 1993a), whereas lonD is essential for sporulation (Gill et al. 1993; Tojo et al. 1993b). The Bacillus subtilis Lon protease is required for the normal transcription of genes controlled by sigmaG, possibly acting by degrading sigmaG. The $B$. subtilis lon mutant, however, has no obvious defect in growth, viability, or sporulation (Schmidt et al. 1994). In humans and yeast, the Lon protease is localized to the mitochondria, where its function is unclear. In yeast, it is essential for growth on nonfermentable carbon sources (Kutejora et al. 1993; Suzuki et al. 1994; Van Dyck et al. 1994; Wang et al. 1994).

We describe here the identification of the C. crescentus Lon homolog and show that it is necessary for removal of the CcrM DNA methyltransferase in the late predivisional cell. The inability to rid the cell of CcrM results in the loss of cell-cycle regulation of the chromosomal methylation state. The lon null mutant strain is morphologically abnormal in ways that are both similar and distinct from that observed for cells in which the chromosome is continuously fully methylated as a result of constitutive transcription of $c c r M$, suggesting that Lon may be important for controlling levels of proteins other than CcrM that are relevant to C. crescentus development. 
Wright et al.

\section{Results}

Isolation of the Caulobacter lon homolog and construction of a lon null mutant strain

Proteolysis is an integral component of the $C$. crescentus cell cycle. In our efforts to identify proteases involved in the turnover of proteins during the cell cycle, we cloned the $C$. crescentus Lon protease homolog. Alignment of the deduced amino acid sequence of the $C$. crescentus Lon homolog with the primary sequence of two representative bacterial Lon proteases shows extensive conservation, particularly around the putative active site serine and the ATP-binding domain (Fig. 2). The C. crescentus Lon protein also shows strong homology to the yeast and human Lon homologs, with $41 \%$ and $38 \%$ identities, respectively.

To understand the role of the Lon protease in the $C$. crescentus cell cycle, we constructed a lon null mutant. A selectable marker (the $\Omega$ cassette) was inserted in the middle of the cloned lon gene, and the resulting construct was integrated into the chromosomal lon locus replacing the wild-type copy (Fig. 3A). To verify the absence of the Lon protease in LS2382, immunoblots were performed using antisera raised against the $E$. coli Lon protease. The antiserum reacted specifically with a 95$\mathrm{kD}$ C. crescentus protein (Fig. 3B, lane 1). This protein was absent in the $C$. crescentus strain LS2382, which contains the $\Omega$ cassette in the lon locus, confirming that this strain is a lon null mutant (Fig. 3B, lane 3). A 4-kb SacI fragment encompassing the lon gene was cloned into the low copy number plasmid pRKlac290 (generating plasmid pRW74). This fragment is sufficient to restore Lon expression in LS2382 as shown by immunoblot analyses of wild-type NA1000/pRW74 and the lon null mutant LS2382/pRW74 (Fig. 3B, lanes 2 and 4, respectively).

\section{Lon mediates CcrM proteolysis \\ during the cell cycle and is essential \\ for cell-cycle-controlled DNA methylation}

Both the CcrM DNA methyltransferase (Stephens et al. 1996) and the McpA chemoreceptor (Alley et al. 1993) are subject to degradation during the $C$. crescentus cell cycle (Fig. 1). To determine whether the Lon protease plays a role in these degradation pathways, we isolated
Figure 2. Amino-acid sequence homology between the C. crescentus, E. coli, and $M$. xanthus Lon proteases. Alignment of the amino acid sequences for the $C$. crescentus (C.c), E. coli (E. coli) (Chin et al. 1988 ), and $M$. xanthus vegetative (lonV) (Tojo et al. 1993a) Lon proteases is shown. A dot represents an identical residue and a dash represents the absence of an amino acid at that site. The boxes highlight the putative ATP-binding domain as determined for E. coli Lon (Chin et al. 1988) and the solid circle indicates the putative active site serine (Amerik et al. 1991). The sequence numbering is for the $M$. xanthus LonV-deduced protein sequence. The alignment was made by use of the computer program Pileup from the GCG sequence package (Devereux et al. 1984).

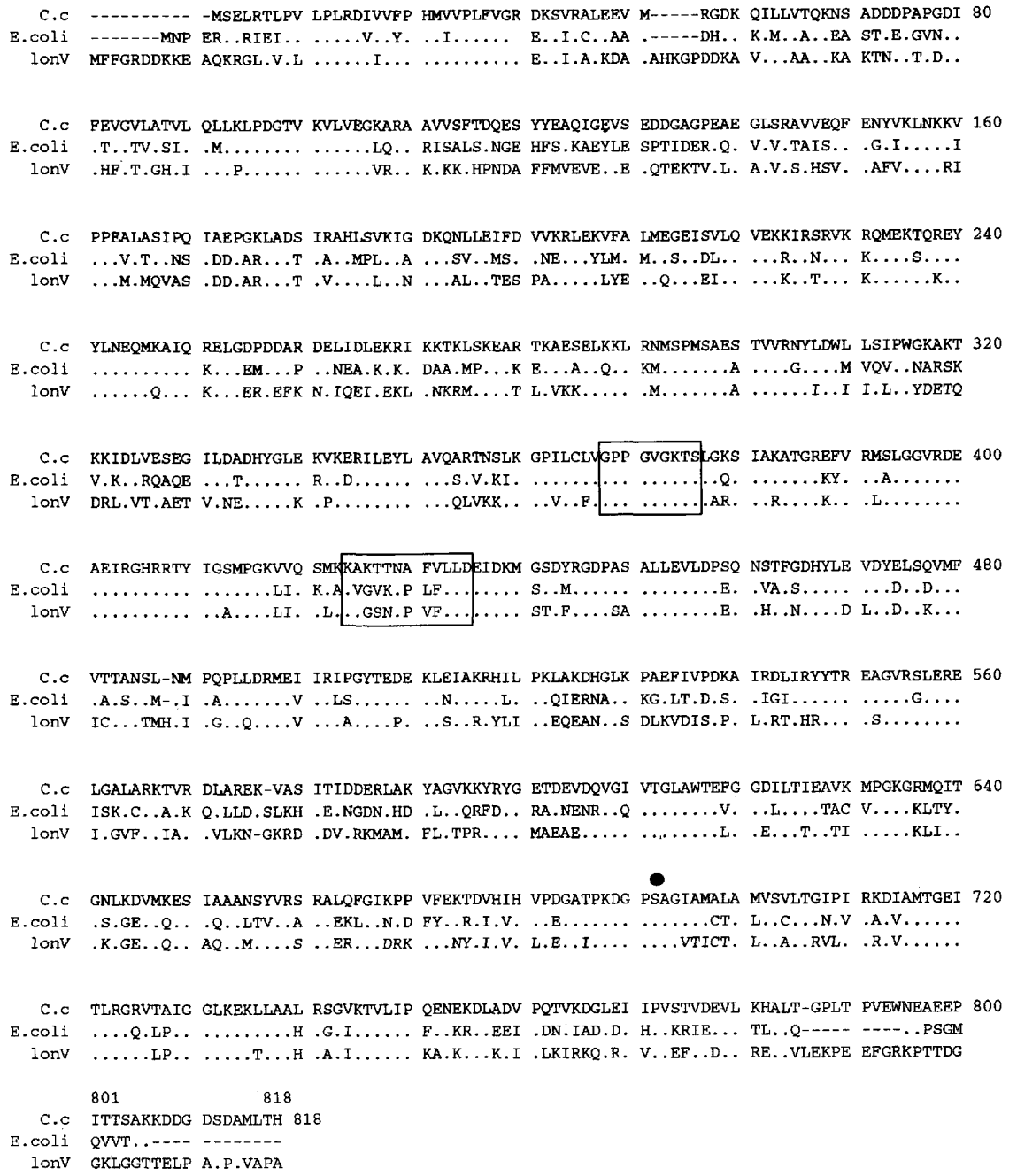

C.C COli -

REVGVIATVL OLLKIPDGTV KVLVEGKARA AVVSFTDOES YYBAOIGEVS BDDGAGPEAE GLSRAVVEOF ENYVKLNKKV 160

coli .T..TV.SI. .M.................. RISALS.NGE HFS.KAEYLE SPTIDER.Q. V.V.TAIS.. .G.I.....

C.C PPEALASIPQ IAEPGKLADS IRAHISVKIG DKQNLLEIFD VVKRLEKVFA LMEGEISVLQ VEKKIRSRVK RQMEKTQREY 240

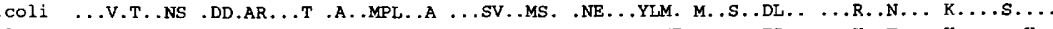

C.C YLNEQMKAIQ RELGDPDDAR DELIDLEKRI KKTKLSKEAR TKAESEIKKL RNMSPMSAES TVVRNYLDWL LSIPWGKAKT 320

.cOli $\ldots \ldots \ldots \ldots$ K...EM...P ..NEA.K.K. DAA.MP...K E...A.Q.. KM....... ........M VQV..NARSK

C.C KKIDLVESEG ILDADHYGLE KVKERILEYL AVQARTNSLK GPICLV GPP GVGKTSLGKS IAKATGREFV RMSLGGVRDE 400

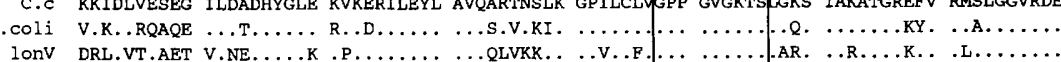

C.C AEIRGHRRTY IGSMPGKVVO SMKKAKTTNA FVLLDEIDKM GSDYRGDPAS ALLEVLDPSO NSTFGDHYLE VDYELSQVMF 480

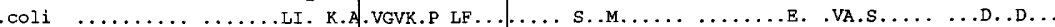

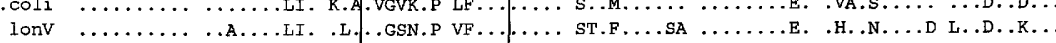

C.C VTTANSL-NM PQPLLDRMEI IRIPGYTEDE KLEIAKRHIL PKLAKDHGLR PAEPIVPDKA IRDLIRYYTR EAGVRSLERE 560

coli .A.S..M-.I .A......V ..LS.............. . . .QIERNA.. KG.LT.D.S. .IGI...........

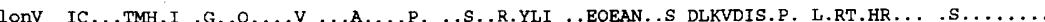

C.C LGALARKTVR DLAREK-VAS ITIDDERLAK YAGVKKYRYG ETDEVDQVGI VTGLAWTEFG GDILTIEAVK MPGKGRMQIT 640

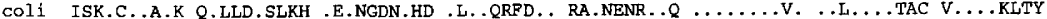

C.C GNLKDVMKES IAAANSYVRS RALQFGIKPP VFEKTDVHIH VPDGATPKDG PSAGIAMALA MVSVLTGIPI RKDIAMTGEI 720

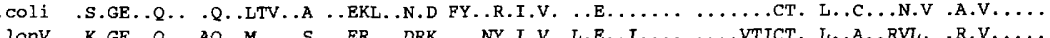

C.C TLRGRVTAIG GLKEKLLAAL RSGVKTVIIP QENEKDLADV PQTVKDGLEI IPVSTVDEVL KHALT-GPLT PVEWNEAEEP 800

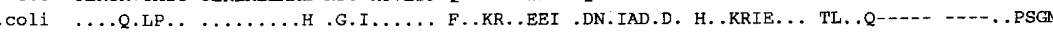

lonV GKLGGTTELP A.P.VAPA 
A

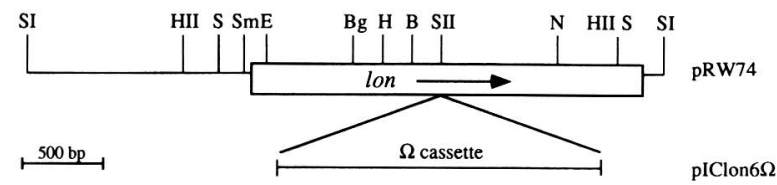

B

$$
\begin{aligned}
& 1 \\
& -\quad 34 \\
& -\quad-95 \mathrm{KDa}
\end{aligned}
$$

Figure 3. Restriction map of the chromosomal region and immunoblot analyses of the C. crescentus lon gene product. $(A)$ Restriction map of the chromosomal region encoding the $C$. crescentus lon gene. The 4-kb SacI fragment (pRW74) was used to complement the lon null mutant LS2382. The $\Omega$ cassette was inserted at the SacII site to disrupt the coding sequence of the lon gene. The restriction sites shown are as follows: (SI) SacI; (HII) HindII; (S) SphI; (Sm) SmaI; (E) EcoRI; (Bg) BglII; (H) HindIII; (B) BamHI; (SII) SacII; (N) NotI. (B) Immunoblot of cell extracts from wild-type $C$. crescentus NA1000 and the lon null mutant, LS2382, probed with the $E$. coli Lon antisera. Crude protein extracts of the C. crescentus strains NA1000 (lane 1), NA1000/pRW74 (lane 2), LS2382 (lane 3), and LS2382/pRW74 (lane 4) were immunoblotted with antisera to $E$. coli Lon as described in Materials and methods. Equal amounts of protein were loaded on each lane. The antibody reacts with a $95-\mathrm{kD} C$. crescentus protein (lane 1) that is absent in LS2382 (lane 3).

swarmer cells from both wild-type NA1000 and LS2382 (Ion null) and allowed these strains to proceed through the cell cycle. Samples were removed at frequent intervals and subjected to immunoblot analysis by use of antibodies against McpA and the CcrM. The immunoblots demonstrate that $\mathrm{CcrM}$ is present throughout the cell cycle in LS2382 (Fig. 4A,B). In contrast, McpA is still subject to proteolysis during the swarmer-to-stalked-cell transition in LS2382 (Fig. 4A,B). These results suggest that Lon is necessary for degradation of CcrM but is not required for degradation of McpA.

The temporal control of the DNA methylation state of the C. crescentus chromosome results from the regulated appearance of the CcrM DNA methyltransferase during the predivisional stage of the cell cycle (Zweiger et al. 1994; Stephens et al. 1996). As CcrM is present throughout the cell cycle in LS2382 (lon null), it is likely that the chromosome remains fully methylated throughout the cell cycle. To test this, the DNA methylation state of a GAnTC site just upstream of the dnaA gene was examined as a function of the cell cycle in NA1000 and LS2382. Samples were removed at frequent intervals from a synchronous culture, and the methylation state of the dnaA chromosomal locus was determined. This demonstrated that in the absence of the Lon protease, this site remains fully methylated throughout the cell cycle (Fig. 4C). Thus, the Lon protease is required for cell-cycle-dependent variation in methylation state at this locus.
The presence of CcrM throughout the cell cycle in the absence of the Lon protease could be caused by either the loss of temporal regulation of $c c r M$ transcription or increased stability of the CcrM protein. We analyzed the ccrM promoter activity in LS2382, which demonstrated

A

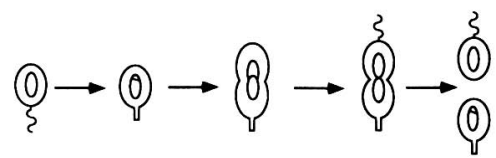

$153045607590 \quad 105120135150165195$ minutes

NA1000

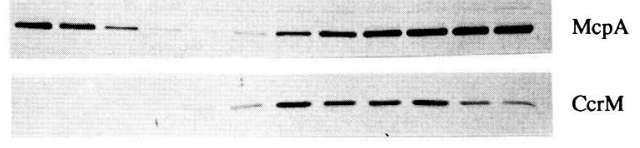

B

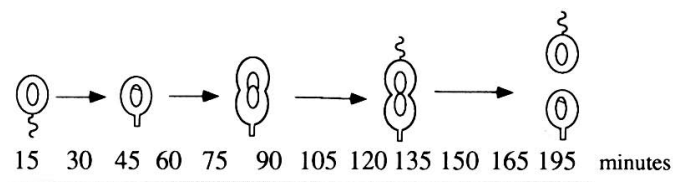

LS2382

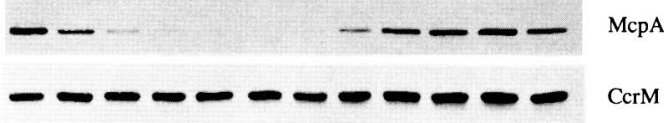

C

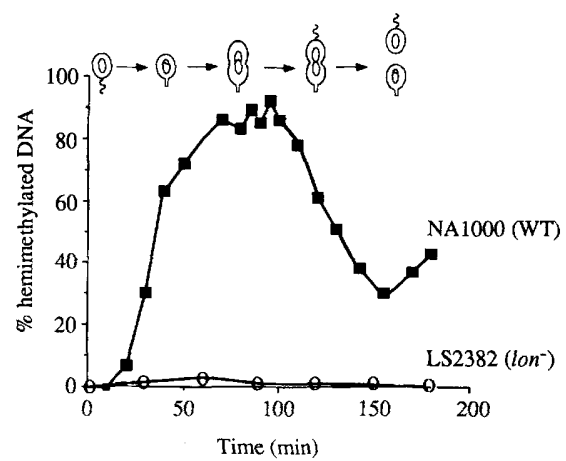

Figure 4. In the absence of the Lon protease, CcrM is present throughout the cell cycle and the chromosome remains fully methylated. Swarmer cells from wild-type NAl000 and the lon null mutant LS2382 were isolated by Ludox density centrifugation (see Material and methods). The cells were resuspended into $\mathrm{M} 2 \mathrm{G}$ minimal media at $30^{\circ} \mathrm{C}$ and allowed to proceed through the cell cycle. Cell-cycle progression was monitored by microscopic analysis and is shown schematically at the top of $A-C$. Cell division of NA1000 occurred at $\sim 160 \mathrm{~min}$ and LS2382 at $\sim 195 \mathrm{~min}$. (A) Immunoblots of protein samples from synchronous NA1000 cultures probed with antisera to McpA or CcrM. (Equal amounts of protein were used in each lane). $(B)$ Immunoblots of protein samples from the synchronous LS2382 culture probed with the McpA and CcrM antibodies. $(C)$ The level of hemimethylated DNA at the $d n a A$ locus during the cell cycle of NA1000 and LS2382. Genomic DNA was isolated at various times, and the DNA methylation state of the dnaA locus was determined as described by Nelson et al. (1984) and Zweiger et al. (1994). Radioactivity levels of the fully and hemimethylated DNA was quantified using the PhosphorImager. Results were plotted as the percentage of hemimethylated DNA relative to the total signal (hemimethylated + full). (a) Wildtype NA1000; (O) the lon null mutant, LS2382. 
that $c c r M$ was still temporally transcribed in LS2382 (data not shown), similar to that observed previously in wild-type cells. Thus, the absence of the Lon protease does not affect cell-cycle-regulated transcription of ccrM. The stability of the CcrM protein was compared in NA1000 and LS2382 by pulse-chase. This showed that the half-life of CcrM had increased dramatically in the absence of the Lon protease (Fig. 5). The half-life of CcrM was $10 \mathrm{~min}$ in NA1000 but was $>120 \mathrm{~min}$ in LS2382, demonstrating that the Lon protease is necessary for rapid proteolysis of CcrM.

To confirm that the Lon protease itself, and not a gene downstream of lon, is required for CcrM proteolysis, we examined the level of CcrM protein through the cell cycle in LS2382 complemented with only the lon-coding region (LS2382/pRW74). We found that CcrM was present only in the predivisional stage of the cell cycle, as in NA1000 (data not shown). Thus, restoring Lon protease activity to LS2382 is sufficient to restore CcrM degradation.

\section{Lon is present throughout the cell cycle}

Because CcrM is present at a specific time in the cell cycle and Lon is required for its degradation, we examined whether the presence of Lon itself might vary as a function of the cell cycle. Immunoblot analysis of samples taken at various times from a synchronous $C$. crescentus culture, by use of the E. coli Lon antibody, showed that the Lon protease is present at similar levels throughout the cell cycle (Fig. 6). The lack of cell-cycleregulation of Lon suggests that the level of CcrM during the cell cycle is determined by a combination of a variable rate of synthesis and constitutive degradation.

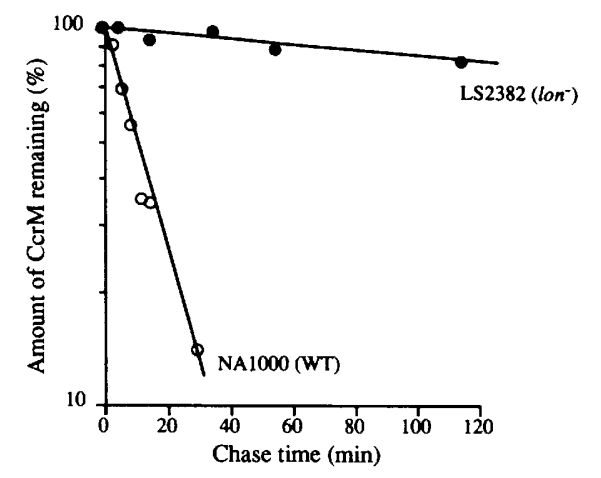

Figure 5. CcrM is a stabilized in LS2382 (lon $\left.{ }^{-}\right)$. The stability of CcrM was determined by pulse-chase in the wild-type C. crescentus strain NA1000 (O) and in LS2382 (lon $\left.{ }^{-}\right)(O)$ as described in Materials and methods. Logarithmically growing NA1000 and LS2382 cultures were pulse-labeled for $5 \mathrm{~min}$ with $\left[{ }^{35} \mathrm{~S}\right]$ methionine and chased with excess methionine, cysteine, and tryptone. Samples were removed at time points thereafter for analysis. CcrM was immunoprecipitated, and the level of labeled CcrM in each sample was quantitated by use of a Molecular Dynamics PhosphorImager. Results are plotted as a percentage of the starting level.

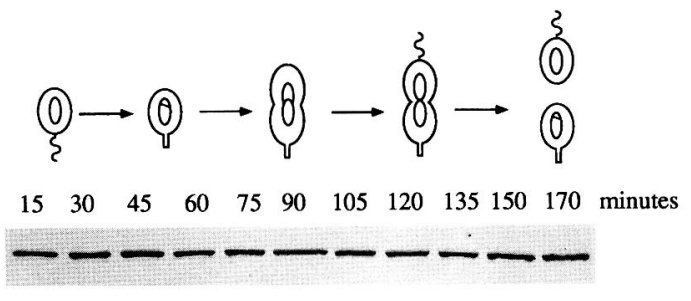

Figure 6. The Lon protease is present throughout the C. crescentus cell cycle. Swarmer cells of wild-type strain NA1000 were released into $\mathrm{M} 2 \mathrm{G}$ medium at $30^{\circ} \mathrm{C}$ and allowed to proceed synchronously through the cell cycle. Cell division occurred at $\sim 160 \mathrm{~min}$. Equal amounts of protein for each sample were immunoblotted with the $E$. coli Lon antibody as described in $\mathrm{Ma}$ terial and methods.

The lon null mutant has developmental abnormalities and the loss of normal timing of initiation of DNA replication

To further understand the role of the Lon protease in $C$. crescentus, we investigated the phenotype of the lon null mutant LS2382. This strain was able to grow under conditions of thermal stress, but in contrast to $E$. coli lon mutants (Gottesman and Maurizi 1992), LS2382 was neither UV sensitive nor mucoid. The growth rate of LS2382 was somewhat slower than wild-type: LS2382 cultures growing logarithmically at $30^{\circ} \mathrm{C}$ in PYE medium have a doubling time of 105 min compared with 90 min for a wild-type culture. It appears that the longer doubling time observed for LS2382 in non-synchronous cultures is caused by a difference in the length of the predivisional stage of the cell cycle. Microscopic analysis of synchronous LS2382 cultures revealed that mutant cells progressed through the cell cycle with kinetics similar to wild-type until the predivisional stage. At this point, the lon null cells stalled and appeared to have difficulties in dividing, although eventually at least half of the cells completed division.

Because CcrM is present throughout the cell cycle in LS2382, we compared the phenotype of LS2382 with the phenotype of a strain in which $\mathrm{ccr} M$ is constitutively expressed. Plasmid pCS226 (Stephens et al. 1996) is a low copy number plasmid containing the $c c r M$ gene transcribed from the xylA promoter, which is constitutively active in cells grown in the presence of xylose (A. Meisenzahl, U. Jenal, and L. Shapiro, pers. comm.). pCS226 was introduced into the NA1000 wild-type strain, generating strain LS2502. When LS2382 (the lon null mutant) and LS2502 (constitutively expressed ccrM) were grown in PYE + xylose $(0.3 \%)$, cells from both strains exhibited morphological abnormalities when compared to wild-type NA1000 cells (Fig. 7A, i, ii, iii). The majority of LS2382 and LS2502 cells were at least two to three times longer than wild type, indicating a significant reduction in the efficiency of cell division in these two strains. There were clear differences, however, between LS2382 and LS2502. Approximately $15 \%$ of the LS2382 cells were very filamentous, with no pinching, 


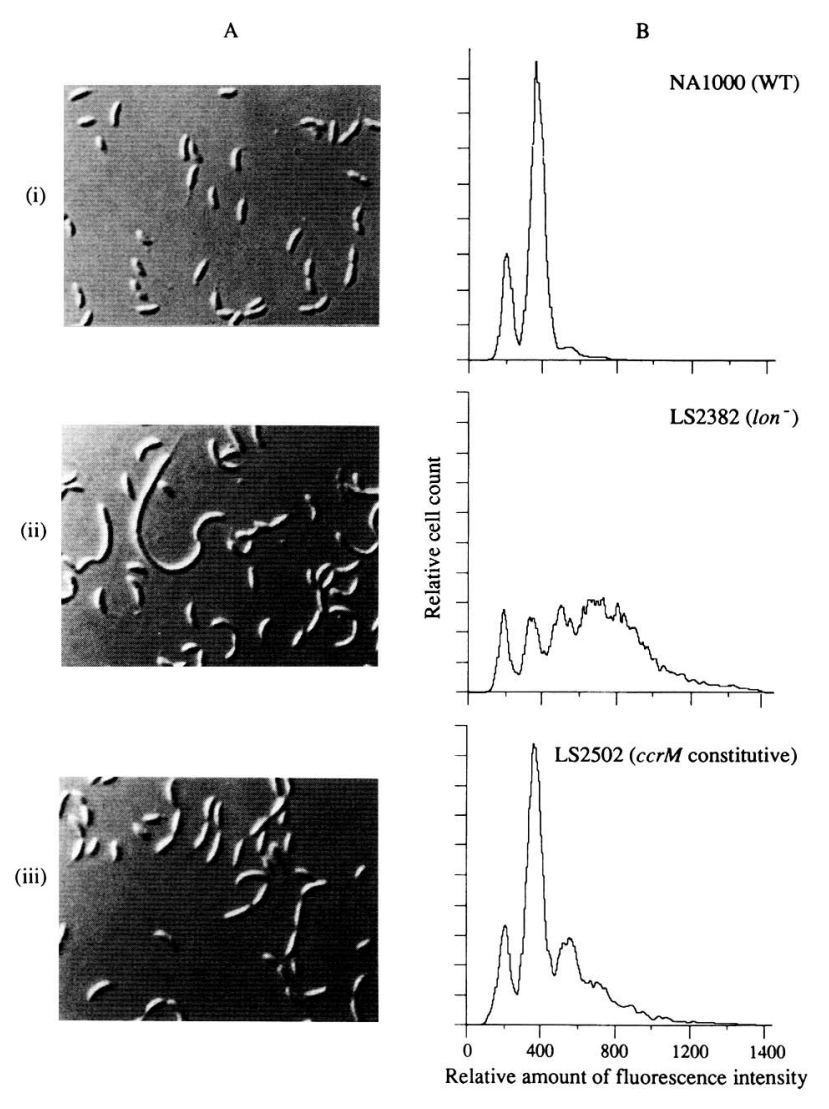

Figure 7. LS2382 (lon null) is morphologically aberrant, has lost normal control of initiation of DNA replication, and is phenotypically similar to a strain in which ccrM is constitutively expressed. Cells from NA1000 (wild type), LS2382 (lon), and LS2502 (ccrM constitutively expressed) were grown in PYE containing xylose $(0.3 \%)$ until $\mathrm{OD}_{600} \sim 0.6$. For flow cytometry analyses, the cells were grown in $25 \mu \mathrm{g} / \mathrm{ml}$ of chloramphenicol for $3 \mathrm{hr}$, fixed in $70 \%$ ethanol, and treated with $10 \mu \mathrm{g} / \mathrm{ml}$ of chromomycin A3. For Nomarski microscopy the cells were spotted onto a polylysine-coated slide and photographed as outlined in Materials and methods. (A) Nomarski micrographs NA1000 (i), LS2382 (ii), and LS2502 (iii). (B) Flow cytometry analyses NA1000 (i), LS2382 (ii), and LS2502 (iii). The vertical axis shows the relative cell count and the horizontal axis the amount of fluorescence intensity.

indicative of inhibition at an early stage of cell division, and the stalks were readily seen by microscopy as being longer and thicker than wild-type (Fig. 7A, i, ii). LS2502 contained filamentous cells four to fivefold longer than wild-type, which exhibited significant pinching, suggesting that division is inhibited at a late stage in these cells. The stalks of this strain were short and often difficult to observe compared to wild-type and LS2382 (Fig. 7A, i, ii, iii).

Control of initiation of DNA replication in LS2382 and LS2502 was compared to wild-type by use of flowcytometry analysis. Flow cytometry has been used previously to determine when the single $C$. crescentus chromosome is replicated during the cell cycle (Winzeler and Shapiro 1995). Logarithmic-phase NA1000, LS2382, and
LS2502 cultures were treated with chloramphenicol, which allows completion of DNA replication, but blocks initiation of DNA replication and prevents cell division. After several hours, cells were fixed and stained for flow cytometry. In a plot of DNA content per cell, two DNA peaks were apparent in NA1000, representing one and two chromosomes per cell. Integration of these peaks shows that $22 \%$ and $70 \%$ of the population had one and two chromosomes, respectively (Fig. 7B, i). The plot of LS2382 showed at least four peaks, indicating the presence of up to four chromosomes per cell. Quantitation of peak areas shows that $10 \%$ had one, $13 \%$ had two, $20 \%$ had three, and $57 \%$ had four or more chromosomes, respectively, in this population (Fig. 7B, ii). In LS2502, only three peaks were clearly apparent. Quantitation demonstrates that within this population, $14 \%$ had one, $49 \%$ had two, $20 \%$ had three, and $17 \%$ had more than three chromosomes, respectively (Fig. 7B, iii). The accumulation of three chromosomes in LS2502 is likely to have arisen in a population of cells that were close to cell division when chloramphenicol was added. Because chromosome replication in the swarmer progeny is normally repressed, whereas the stalked progeny immediately reinitiates replication (Brun et al. 1994), we speculate that the third chromosome arises from premature initiation of replication in the stalked pole of the predivisional cell. In the presence of chloramphenicol (to block cell division), this round of replication is then completed. In the lon null mutant, there was an even greater relaxation of control of the initiation of DNA replication, as more than half of the population contained at least four chromosomes.

\section{Discussion}

We have demonstrated that restriction of the CcrM DNA methyltransferase to the predivisional cell, a requirement for the normal progression of the C. crescentus cell cycle, is dependent on the Lon protease. The level of CcrM protein is controlled through transcription and protein instability, to ensure that CcrM activity, and consequently, DNA methylation, occurs only in the predivisional cell (Zweiger et al. 1994; Stephens et al. 1995). In the absence of the Lon protease, $c c r M$ is still transcriptionally regulated, but the CcrM protein is dramatically stabilized, with a half-life at least 12 -fold longer than in wild-type. The resulting presence of CcrM throughout the cell cycle leads to persistently fully methylated chromosomes. Ion null cells are unable to divide normally, have aberrant shapes, and lose, to a significant extent, the ability to regulate initiation of DNA replication during the cell cycle.

The C. crescentus lon gene encodes a predicted protein of 799 amino acids that shows extensive homology to other members of the Lon protease family. Lon is not essential for viability, but the morphology and growth pattern of the lon null mutant suggests that the normal progression of the cell cycle is affected. We have identified an important target for the Lon protease pathway in C. crescentus, the CcrM DNA methyltransferase. The 
constitutive presence of Lon during the C. crescentus cell cycle suggests a simple model accounting for the transient appearance of CcrM in the predivisional cell. Upon induction of $c c r M$ transcription, the rate of synthesis becomes greater than the rate of degradation, such that CcrM accumulates. ccrM transcription shuts off prior to cell division, and the level of CcrM protein declines rapidly as a result of continous Lon-dependent proteolysis, so that after separation of the progeny cells, only very low levels of CcrM are detected. We cannot rule out the possibility that at different stages of the cell cycle, CcrM is transiently protected from degradation, possibly through interaction with another protein. The level of CcrM protein in the predivisional cell, however, must be tightly regulated to ensure that there is sufficient enzyme to completely remethylate the newly replicated chromosomes, without exceeding the capacity of Lon to remove it prior to cell division. A similar type of regulation has been demonstrated in $E$. coli for the SulA protein (Mizusawa and Gottesman 1983; Schoemaker et al. 1984; Gottesman and Maurizi 1992). On induction of the SOS response, sulA transcription is activated, and the resulting SulA protein interacts with FtsZ to cause transient inhibition of cell division (Huisman et al. 1984; Jones and Holland 1985). SulA is highly unstable and accumulates only when transcription is activated. When sulA transcription ceases, because of inactivation of the SOS response, the SulA protein is degraded rapidly in a Lon-dependent manner to restore cell division (Huisman et al. 1984; Schoemaker et al. 1984).

How is CcrM targeted for proteolysis in a Lon-dependent manner? Although we have demonstrated that rapid degradation of CcrM requires the Lon protease, it is possible that additional proteins, such as chaperones, are also involved in specific proteolysis of CcrM. In E. coli, both the Lon protease and the DnaK chaperone are required for the degradation of the mutant form of alkaline phosphatase phoA61 (Sherman and Goldberg 1992). Regardless, the CcrM protein presumably contains a signal that tags it to be rapidly degraded in a Lon-dependent manner. In $E$. coli, the signals that target proteins for degradation by Lon are not well understood. Dervyn et al. (1990) deleted the Carboxy-terminal half of SulA and found that it was still subject to Lon-dependent proteolysis. There are no amino acid sequence motifs that appear to be conserved among known short-lived proteins in $E$. coli, suggesting that recognition by Lon may depend on structural elements rather than specific sequences.

The restriction of DNA methylation activity to the predivisional cell is important for normal progression of the $C$. crescentus cell cycle. When CcrM is present throughout the cell cycle, either through constitutive expression of $c c r M$ (LS2502) or increased stability of the CcrM protein (LS2382), the chromosome is persistently fully methylated. Both LS2382 and LS2502 exhibit defects in cell division and the timing of initiation of DNA replication, suggesting that the abnormal phenotypic properties of the lon null mutant are at least partially caused by the altered methylation state of the chromosome. Nevertheless, there are significant differences be- tween LS2382 and LS2502. For instance, LS2382 has long stalks, whereas the stalks in LS2502 are short compared to wild-type cells. Thus, it is likely that there are additional unidentified proteins whose degradation is Londependent and that are responsible for the differences observed between LS2382 and LS2502. We suspect that the $C$. crescentus Lon protease has multiple roles analogous to the $E$. coli Lon protease, which under physiological conditions is required for the degradation of several short-lived proteins and under thermal stress removes damaged proteins from the cell (for review, see Gottesman and Maurizi 1992).

Previous results have suggested that the $C$. crescentus Lon protease is preferentially synthesized in the stalked pole of the predivisional cell and the progeny stalked cell (Reuter and Shapiro 1987). In addition, there is a two-fold bias of the level of the Lon protease in the stalked cell compared to the swarmer cell directly after cell division (data not shown). This slight bias could exist to ensure that residual CcrM protein is removed thoroughly from the stalked cell (which initiates DNA replication) in order to prevent any DNA methyltransferase activity in this cell type. In $E$. coli, initiation of DNA replication typically occurs on a fully methylated origin, but is prevented on a hemimethylated template /Ogden et al. 1988; Boye and Lobner-Oleson 1990; Campbell and Kleckner 1990). The C. crescentus chromosomal origin of replication contains several CcrM methylation sites (Marczynski and Shapiro 1992), whose regulatory significance is not yet known. The defects in control of replication initiation during the cell cycle in LS2382 and LS2502 are consistent with a role for DNA methylation state influencing the initiation of DNA replication. Since initiation of DNA replication in C. crescentus occurs immediately in the progeny stalked cell, it would be critical to ensure that there is no CcrM remaining after division, particularly in the stalked progeny. The removal of CcrM may also be important to ensure that as yet unidentified regulatory sites, other than the replication origin, retain a period of hemimethylation during the cell cycle, allowing normal progression of the cell cycle, and ultimately cell division.

Protein degradation is emerging as an important contributor to cellular regulation and function in bacteria. We have demonstrated that the Lon protease is involved in degradation of the CcrM DNA methyltransferase, a protein that is required for temporally controlled DNA methylation and the progression of the cell cycle in $C$. crescentus. It is becoming evident that the identification of proteases responsible for modulating the stability of key regulatory proteins in bacteria, as well as eukaryotes, is necessary to further our understanding of cell cycle control circuits.

\section{Materials and methods}

Bacterial strains and growth conditions

Routine DNA manipulations and growth of $E$. coli strains were performed essentially as described by Sambrook et al. (1989). 
Bacterial strains and plasmids used in this study are listed in Table 1. C. crescentus strains were grown at $30^{\circ} \mathrm{C}$ in either PYE complex medium or M2G minimal media supplemented with kanamycin $(5 \mu \mathrm{g} / \mathrm{ml})$, tetracycline $(1 \mu \mathrm{g} / \mathrm{ml})$, ampicillin $(10 \mu \mathrm{g} /$ $\mathrm{ml})$, nalidixic acid $(20 \mu \mathrm{g} / \mathrm{ml})$, streptomycin $(5 \mu \mathrm{g} / \mathrm{ml})$, or spectinomycin $(25 \mu \mathrm{g} / \mathrm{ml})$ as required. Plasmids were mobilized from the E. coli strain S17-1 into C. crescentus by conjugation (Ely 1991).

\section{Cloning the Caulobacter lon homolog}

Two primers were designed for PCR based on regions of homology between the $M$. xanthus LonV, LonD (Tojo et al 1993a,b) and the E. coli Lon proteases (Chin et al. 1988): Lon 1 (5'-CTGTGCCTCGTCGGTCCCCCGGGC-3') and LonIII (5'-CTGCTCCGGATCCAGCAGCTCGAG-3'). These primers were used with genomic DNA from C. crescentus NA1000 as template in PCR to amplify a 300-bp product. The product was cloned into pBluescript and sequenced to confirm that it encoded a homolog of the Lon protease family. The PCR product was used to screen a C. crescentus XZAP library (Brun and Shapiro 1992) at high stringency. Four positive clones were isolated: plon2, plon4, plon5, and plon6. Sequence analyses revealed that these clones contained only part of the lon gene. The full length gene was cloned using a plasmid recovery technique. The 2.0 -kb insert from plon 2 was ligated into the narrow host range vector pDelta creating pDlon2. pDlon 2 was introduced into NA1000, and integrants at the lon locus were selected by growth on kanamycin. The genomic DNA of the resulting strain was digested with EcoRI and religated, creating pELl. pELl contains the pDelta vector with $8 \mathrm{~kb}$ of chromosomal DNA extending from the lon locus to the nearest EcoRI site encompassing the lon gene.

Small fragments covering the lon gene were subcloned into the vector pBluescript. DNA sequencing was performed by use of either the $\Delta$-Taq DNA sequencing kit (Amersham) or an automated DNA sequencer (Applied Systems, Model 373A, PAN facility, Stanford University) with either single or doublestranded DNA templates. All sequence analyses were performed with the GCG sequence package from the University of Wisconsin (Devereux et al. 1984). The C. crescentus lon gene has been assigned the Genbank accession no. U56652.

\section{Other plasmid and strain constructions}

The lon null mutant, LS2382, was constructed by ligating the 4.0 -kb insert from plon6 into the narrow host range vector pIC20R creating pIClon6. The $\Omega$ cassette from the plasmid pHP45 $\Omega$, encoding a spectinomycin/streptomycin resistance

Table 1. Strains and plasmids

\begin{tabular}{|c|c|c|}
\hline Strain/plasmid & Description & $\begin{array}{l}\text { Reference/ } \\
\text { source }\end{array}$ \\
\hline \multicolumn{3}{|l|}{ Escherichia coli } \\
\hline $\mathrm{DHIOB}$ & cloning strain & GIBCO BRL \\
\hline TGI & cloning strain & T.J. Gibson (unpubl.) \\
\hline S17 & RP4-2, Tc::Mu Km::Tn7 & (Simon et al. 1983) \\
\hline \multicolumn{3}{|l|}{ C. crescentus } \\
\hline NA1000 & synchronizable derivative of wild-type strain & (Evinger and Agabian 1977) \\
\hline LS107 & NA1000 $\Delta b l a 6$ & M.R.K. Alley (unpubl.) \\
\hline LS1837 & NA1000 $\Delta$ bla6 lon:: $\Omega$ & this study \\
\hline LS2382 & NA1000 lon:: $\Omega$ & this study \\
\hline LS2502 & NA1000 pCS226 & this study \\
\hline \multicolumn{3}{|l|}{ Plasmids } \\
\hline pBluescript & $A m p^{R}$ & Stratagene \\
\hline pIC20R & $A m p^{R}$ & (Marsh et al. 1984) \\
\hline pDelta & suicide vector, $A m p^{R}, \operatorname{Kan}^{R}, \operatorname{Tet}^{R}, \operatorname{Suc}^{S}$ & GIBCO BRL \\
\hline pRKlac290 & pRK290 derivative, lac $Z$ transcriptional fusion vector & (Gober and Shapiro 1992) \\
\hline $\mathrm{pHP} 45 \Omega$ & $\begin{array}{l}\text { pBR322 derivative containing the } \Omega \text { cassette encoding for spectinomycin and } \\
\text { streptomycin resistance }\end{array}$ & (Prentki and Krisch 1984) \\
\hline plon2 & 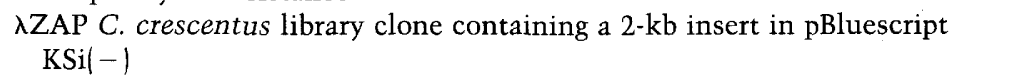 & this study \\
\hline plon 4 & $\begin{array}{l}\text { גZAP C. crescentus library clone containing a } 2-\mathrm{kb} \text { insert in pBluescript } \\
\quad \mathrm{KSi}(-)\end{array}$ & this study \\
\hline plon5 & $\begin{array}{l}\text { גZAP C. crescentus library clone containing a } 2-\mathrm{kb} \text { insert in pBluescript } \\
\mathrm{KSi}(-)\end{array}$ & this study \\
\hline plon6 & $\begin{array}{l}\text { גZAP C. crescentus library clone containing a } 4-\mathrm{kb} \text { insert in pBluescript } \\
\mathrm{KSi}(-)\end{array}$ & this study \\
\hline pDlon2 & plon2 insert, end-filled and ligated into pDelta & this study \\
\hline pIClon 6 & 4-kb insert from plon6 ligated into $\mathrm{pIC} 20 \mathrm{R}$ & this study \\
\hline pIClon $6 \Omega$ & $\Omega$ cassette inserted into the SacII site of pIClon 6 & this study \\
\hline pEL1 & $\begin{array}{l}\text { pDelta vector containing } 8-\mathrm{kb} \text { fragment encompassing the lon gene } \\
\text { generated from the NA1000 chromosome }\end{array}$ & this study \\
\hline pCS226 & xylA::ccrM in pRKlac290 & (Stephens et al. 1996) \\
\hline pRW74 & 4.0-kb SacI fragment from pELl containing the full-length lon gene & this study \\
\hline pDnaA & $\begin{array}{l}\text { 530-bp XbaI-SalI fragment containing the end of the rpsT gene and the } \\
\text { beginning of the } d \text { naA gene in pBluescript SKII }-1\end{array}$ & G. Zweiger (unpubl.) \\
\hline
\end{tabular}


determinant flanked by transcriptional and translational stop signals, was ligated into the single SacII site of pIClon6 /which is located in the middle of the lon gene), creating pIClon $6 \Omega$. pIClon $6 \Omega$ was introduced into the strain LS107, and integrants at the lon locus were selected for by growth on spectinomycin/ streptomycin. The resulting strain contained one functional and one disrupted copy of the lon gene separated by plasmid sequence containing the $\operatorname{sac} B$ gene. Subsequent growth of this strain on 3\% sucrose (Gay et al. 1985), followed by streptomycin and spectinomycin, selected for the recombination event that resulted in the disrupted copy of the lon gene remaining in the chromosome to generate the strain LS1837. A $\phi C r 30$ transducing lysate was made of LS1837 (Ely 1991) and was transduced into wild-type C. crescentus strain NA1000, creating LS2382. Southern blot analyses were performed to confirm that the lon gene was disrupted as predicted and that there was no wild-type copy in the chromosome of LS2382.

The plasmid pRW74 was constructed by ligating the 4-kb SacI fragment from pELl, encompassing the lon gene, into the low copy number vector pRKlac290 (Gober and Shapiro 1992). pRW74 was introduced into NA1000 and LS2382 by conjugation.

LS2502 was constructed using the plasmid pCS226 /Stephens et al. 1996). pCS226 contains the xylA promoter (A. Meisenzahl, U. Jenal, and L. Shapiro, pers. comm.) transcriptionally fused to the $c c r M$ gene in the low copy number plasmid pRKlac290. pCS226 was mated into NA1000 and $\mathrm{Tet}^{\mathrm{R}}$ strains were isolated generating the strain LS2502. To constitutively transcribe $c c r M$ from the xylA promoter, LS2502 was grown in PYE + xylose $(0.3 \%)$ with no glucose, and to repress this promoter, LS2502 was grown in PYE containing glucose $(0.2 \%)$ without xylose.

\section{Synchronization of Caulobacter}

C. crescentus strains grown in minimal $\mathrm{M} 2 \mathrm{G}$ medium were synchronized by isolating the swarmer population with Ludox density gradient centrifugation (Evinger and Agabian 1977). Swarmer cells were resuspended into M2G and allowed to proceed through the cell cycle at $30^{\circ} \mathrm{C}$. For NA1000, the swarmer population was $>95 \%$ pure, as determined by microscopy. The population of LS 2382 was $\sim 80 \%$ pure, the remainder being cells of two to three times the length of wild-type.

\section{Immunoblotting}

C. crescentus cells in logarithmic growth $(1 \mathrm{ml})$ were pelleted by centrifugation and resuspended into SDS-sample buffer and boiled for $5 \mathrm{~min}$. Protein concentrations for each sample were determined by $A_{280}$ analysis and, unless otherwise stated, equal amounts of protein were loaded for each gel. The samples were electrophoresed either in $10 \%$ or $12 \%$ SDS-polacrylamide gels, and the separated proteins were transferred to Immobilon-P membrane (Millipore). Immunoblot analyses were performed as outlined in Sambrook et al. (1989) by use of either the primary antibody $\alpha-\mathrm{CcrM}$ at a $1 / 5000$ dilution or $\alpha-\mathrm{McpA}$ at a $1 / 10000$ dilution or $\alpha$-Lon at a 1/500 dilution (kindly provided by $\mathrm{A}$. Markovitz, University of Chicago, IL) with HRP-conjugated goat anti-rabbit IgG, Boehringer Mannheim $\{1: 10,000$ dilution) as the secondary antibody. The immunoblot was developed using the Renaissance Chemiluminescence kit (Dupont NEN) following the manufacturer's instructions.

\section{Determination of CcrM half-life}

Mixed cell cultures were grown to an $\mathrm{OD}_{600} 0.4$ in M2G minimal media. Ten milliliters of culture was labeled for $2 \mathrm{~min}$ with $20 \mu \mathrm{Ci} / \mathrm{ml}\left[{ }^{35} \mathrm{~S}\right]$ methionine (Trans-label, ICN) and chased with unlabeled methionine $(2 \mathrm{~mm})$, cysteine $(2 \mathrm{~mm})$, and $(0.2 \%)$ tryptone. After the addition of the chase mixture, samples were removed from NA1000 cultures at 0,3,6,9,12, 15, and $30 \mathrm{~min}$ and for LS2382 cultures at $0,5,15,35,55$, and $115 \mathrm{~min}$. The cells were centrifuged for $1 \mathrm{~min}$, the supernatant was removed, and the cell pellet was placed immediately onto dry ice. The labeled CcrM protein was immunoprecipitated by use of the CcrM antisera (1/500 dilution) from equal amounts of labeled total cellular protein as previously described by Jenal and Shapiro (1996). Quantitation was performed by use of a Molecular Dynamics PhosphorImager with ImageQuant software. The results were plotted as a percentage of labeled CcrM remaining compared to the first time-point signal.

\section{Nomarski microscopy}

Cells from logarithmic-phase growth in rich PYE media containing xylose $(0.3 \%)$ were placed on a polylysine $(0.1 \% \mathrm{wt} / \mathrm{vol})-$ coated slide, allowed to settle for $15 \mathrm{~min}$, and washed several times with distilled water. The cells were photographed without staining on a Zeiss Axiophot microscope with DIC (Nomarski) optics.

\section{Flow cytometry}

NA1000 and LS2382 were grown overnight in PYE containing $0.3 \%$ xylose, and LS2502 was grown in PYE containing $0.3 \%$ xylose and $1 \mu \mathrm{g} / \mathrm{ml}$ of tetracycline. Cultures were pelleted by gentle centrifugation and resuspended in PYE $(3 \mathrm{ml})$ containing xylose $(0.3 \%)$ without antibiotic at an $\mathrm{OD}_{600} \sim 0.1$. At $\mathrm{OD}_{600}$ $\sim 0.6,25 \mu \mathrm{g} / \mathrm{ml}$ of chloramphenicol was added and the cells were grown for another $3 \mathrm{hr}$ at $30^{\circ} \mathrm{C}$. Cells were fixed and stained for flow cytometry analyses as described by Winzeler and Shapiro (1995). For each flow cytometry experiment, the DNA content was measured in a population of 10,000 cells using a Becton Dickson FACStar Plus machine with excitation at $458 \mathrm{~nm}$, and the fluorescence was measured at $495 \mathrm{~nm}$. The data was collected and analyzed using the FACS/DESK software (Stanford University, Stanford, CA).

\section{Analysis of DNA methylation state}

Cultures were synchronized in M2G minimal media, as described earlier. Swarmers were released into M2G media at $\mathrm{OD}_{600} \sim 0.3-0.4$ and were allowed to proceed through the cell cycle. Samples were removed at various times, and genomic DNA was isolated by use of the Puregene DNA isolation kit (Gentra Systems). The DNA methylation state of a representative site upstream of the $d n a A$ gene was determined by use of an overlapping HinfI-HindII site. For a detailed explanation on this DNA methylation assay, see Nelson et al. (1984) and Zweiger et al. (1994). Southern blots of HindII digested genomic DNA were probed with a randomly labeled (T7 QuickPrime kit, Pharmacia) 420-bp HindII fragment from pDnaA. This fragment is on one side of the overlapping HinfI- HindII site, and therefore, the level of hemimethylated DNA at that site can be quantitated. The bands representing fully $(820 \mathrm{bp}$ ) and hemimethylated $(420$ bp) DNA on the Southern blots were quantitated by use of a Molecular Dynamics PhosphorImager with ImageQuant software. As the probe only detects one of the two hemimethylated products, the signal from the hemimethylated band was multiplied by two, and the \% hemimethylated DNA was determined as a percentage of the total signal. 


\section{Acknowledgments}

We thank Kim Quon and Jeff Skerker for help with the microscopy, Elizabeth Winzeler for help with the FACS analyses, and Urs Jenal and members of the Shapiro laboratory for their helpful comments and critical reading of the manuscript. This work was supported by the National Institutes of Health grants GM32506 and GM-52426. R.W. was supported by the Katharine McCormick and Dean's Fund from Stanford University.

The publication costs of this article were defrayed in part by payment of page charges. This article must therefore be hereby marked "advertisement" in accordance with 18 USC section 1734 solely to indicate this fact.

\section{References}

Alley, M.R., J.R. Maddock, and L. Shapiro. 1992. Polar localization of a bacterial chemoreceptor. Genes \& Dev. 6: 825-836.

- 1993. Requirement of the carboxyl terminus of a bacterial chemoreceptor for its targeted proteolysis. Science 259: 1754-1757.

Amerik, A.Y., V.K. Antonov, A.E. Gorbalenya, S.A. Kotova, T.V. Rotanova, and E.V. Shimbarevich. 1991. Site-directed mutagenesis of La protease: A catalytically active serine residue. FEBS Lett. 287: 211-214.

Boye, E. and A. Lobner-Olesen. 1990. The role of dam methyltransferase in the control of DNA replication in E. coli. Cell 62: 981-989.

Brun, Y.V. and L. Shapiro. 1992. A temporally controlled $\sigma$-factor is required for polar morphogenesis and normal cell division in Caulobacter. Genes \& Dev. 6: 2395-2408.

Brun, Y.V., G. Marczynski, and L. Shapiro. 1994. The expression of asymmetry during Caulobacter cell differentiation. Annu. Rev. Biochem. 63: 419-450.

Campbell, J.L. and N. Klechner. 1990. E. coli oriC and the dnaA gene promoter are sequestered from dam methylation following the passage of the chromosomal replication fork. Cell 62: 967-979.

Chin, D.T., S.A. Goff, T. Webster, T. Smith, and A.L. Goldberg. 1988. Sequence of the lon gene in Escherichia coli. I. Biol. Chem. 263: 11718-11728.

Dervyn, E., D. Canceill, and O. Huisman. 1990. Saturation and specificity of the Lon protease of Escherichia coli. I. Bacteriol. 172: 7098-7103.

Devereux, D., P. Haeberli, and O. Smithies. 1984. A comprehensive set of sequence analysis program for the VAX. $\mathrm{Nu}$ cleic Acids Res. 12: 387-395.

Ely, B. 1991. Genetics of Caulobacter crescentus. Methods Enzymol. 204: 372-384.

Evinger, M. and N. Agabian. 1977. Envelope-associated nucleoid from Caulobacter crescentus stalked and swarmer cells. J. Bacteriol. 132: 294-301.

Gay, P., D. Le Coq, M. Steinmetz, T. Berkelman, and C.I. Kado. 1985. Positive selection procedure for entrapment of insertion sequence elements in gram-negative bacteria. I. Bacteriol. 164: 918-921.

Gill, R.E., M. Karlok, and D. Benton. 1993. Myxococcus xanthus encodes an ATP-dependent protease which is required for developmental gene transcription and intercellular signaling. I. Bacteriol. 175: 4538-4544.

Gober, J.W. and L. Shapiro. 1992. A developmentally regulated Caulobacter flagellar promoter is activated by 3 ' enhancer and IHF binding elements. Mol. Biol. Cell 3: 913-926.

Goldberg, A.L., R.P. Moerschell, C.H. Chung, and M. Maurizi. 1994. ATP-dependent protease La (Lon) from Escherichia coli. Methods Enzymol. 244: 350-375.
Gottesman, S. and M.R. Maurizi. 1992. Regulation by proteolysis: Energy-dependent proteases and their targets. Microbiol. Rev. 56: 592-621.

Gottesman, S., M.E. Gottesman, J.E. Shaw, and L. Pearson. 1981. Protein degradation in E. coli: The lon mutation and bacteriophage lambda $\mathrm{N}$ and $\mathrm{CII}$ protein stability. Cell 24: 225-233.

Huisman, O., R. D'Ari, and S. Gottesman. 1984. Cell division control in Escherichia coli: Specific induction of the SOS SfiA protein is sufficient to block septation. Proc. Natl. Acad. Sci. 81: 4490-4494.

Jenal, U. and L. Shapiro. 1996. Cell cycle-controlled proteolysis of a flagellar motor protein that is asymmetrically distributed in the Caulobacter predivisional cell. EMBO $\mathrm{f}$. (in press).

Jones, C. and I.B. Holland. 1985. Role of the SulB (FtsZ) protein in division inhibition during the SOS response in Escherichia coli: FtsZ stabilizes the inhibitor SulA in maxicells. Proc. Natl. Acad. Sci. 82: 6045-6049.

King, R.W., P.K. Jackson, and M.W. Kirschner. 1994. Mitosis in transition. Cell 79: 563-571.

Kutejova, E., G. Durcova, E. Surovkova, and S. Kuzela. 1993. Yeast mitochondrial ATP-dependent protease: Purification and comparison with the homologous rat enzyme and the bacterial ATP-dependent protease. FEBS Lett. 329: 47-50.

Marczynski, G. and L. Shapiro. 1992. Cell cycle control of a cloned chromosomal origin of replication from Caulobacter crescentus. J. Mol. Biol. 225: 959-977.

Marsh, J.L., M. Erfle, and E.J. Wykes. 1984. The pIC plasmid and phage vectors with versatile cloning sites for recombinant selection by insertional inactivation. Gene 32: 481-485.

Mizusawa, S. and S. Gottesman. 1983. Protein degradation in Escherichia coli: The lon gene controls the stability of SulA protein. Proc. Nat1. Acad. Sci. 80: 358-362.

Nelson, M., C. Christ, and I. Schildkraut. 1984. Alteration of apparent restriction endonuclease recognition specificity by DNA methlyase. Nucleic Acids Res. 12: 5165-5173.

Ogden, G.B., M.J. Pratt, and M. Schaechter. 1988. The replicative origin of the $E$. coli chromosome binds to cell membranes only when hemimethylated. Cell 54: 127-135.

Prenki, H. and H.M. Krisch. 1984. In vitro insertional mutagenesis with a selectable DNA fragment. Gene 29: 303-313.

Quon, K.C., G.T. Marczynski, and L. Shapiro. 1996. Cell cycle control by an essential bacterial two-component signal transduction protein. Cell 84: 1-20.

Reuter, S.H. and L. Shapiro. 1987. Asymmetric segregation of heat-shock proteins upon cell division in Caulobacter crescentus. I. Mol. Biol. 194: 653-662.

Sambrook, J., E.F. Fritsch, and T. Maniatis. 1989. Molecular cloning. A laboratory manual. Cold Spring Harbor Laboratory Press, Cold Spring Harbor, NY.

Schmidt, R., A.L. Decatur, P.N. Rather, C.P. Moran Jr., and R. Losick. 1994. Bacillus subtilis Lon protease prevents inappropriate transcription of genes under the control of the sporulation transcription factor $\sigma^{\mathrm{G}}$. J. Bacteriol. 176: 65286537.

Schoemaker, J.M., R.C. Gayda, and A. Markovitz. 1984. Regulation of cell division in Escherichia coli: SOS induction and cellular location of the SulA protein, a key to Lon-associated filamentation and death. I. Bacteriol. 158: 551-561.

Sherman, M.Y. and A.L. Goldberg. 1992. Involvement of the chaperonin dnaK in the rapid degradation of a mutant protein in Escherichia coli. EMBO J. 11: 71-77.

Simon, R., U. Prieffer, and A. Puhler. 1983. A broad host range mobilization system for in vivo genetic engineering: Transposon mutagenesis in gram negative bacteria. Biotechnology 


\section{Wright et al.}

1: 784-790.

Stephens, C.M., G. Zweiger, and L. Shapiro. 1995. Coordinate cell cycle control of a Caulobacter DNA methyltransferase and the flagellar genetic hierarchy. J. Bacteriol. 177: 16621669.

Stephens, C., A. Reisenauer, R. Wright, and L. Shapiro. 1996. A cell cycle-regulated bacterial DNA methyltransferase is essential for viability. Proc. Natl. Acad. Sci. 93: 1210-1214.

Suzuki, C.K., K. Suda, N. Wang, and G. Schatz. 1994. Requirement for the yeast gene lon in intramitochondrial proteolysis and maintenance of respiration. Scienc 264: 273-276.

Tojo, N., S. Inouye, and T. Komano. 1993a. Cloning and nucleotide sequence of the Myxococcus xanthus lon gene: Indispensability of lon for vegetative growth. I. Bacteriol. 175: 2271-2277.

. 1993b. The lonD gene is homologous to the lon gene encoding an ATP-dependent protease and is essential for the development of Myxococcus xanthus. I. Bacteriol. 175: 4545-4549.

Torres-Cabassa, A.S. and S. Gottesman. 1987. Capsule synthesis in Escherichia coli $\mathrm{K}-12$ is regulated by proteolysis. I. Bacteriol. 169: 981-989.

Van Dyck, L., D.A. Pearce, and F. Sherman. 1994. PIM1 encodes a mitochondrial ATP-dependent protease that is required for mitochondrial function in the yeast Saccharomyces cerevisiae. I. Biol. Chem. 269: 238-242.

Van Melderen, L., P. Bernard, and M. Couturier. 1994. Londependent proteolysis of CcdA is the key control for activation of CcdB in plasmid-free segregant bacteria. Mol. Microbiol. 11: 1151-1157.

Wang, N., M.R. Maurizi, L. Emmeert-Buck, and S. Gottesman. 1994. Synthesis, processing and localization of human Lon protease. J. Biol. Chem. 269: 29308-29313.

Winzeler, E. and L. Shapiro. 1995. Use of flow cytometry to identify a Caulobacter 4.5S RNA temperature-sensitive mutant defective in the cell cycle. I. Mol. Biol. 251: 346-365.

Zweiger, G., G. Marczynski, and L. Shapiro. 1994. A Caulobacter DNA methyltransferase that functions only in the predivisisional cell. I. Mol. Biol. 235: 472-485. 


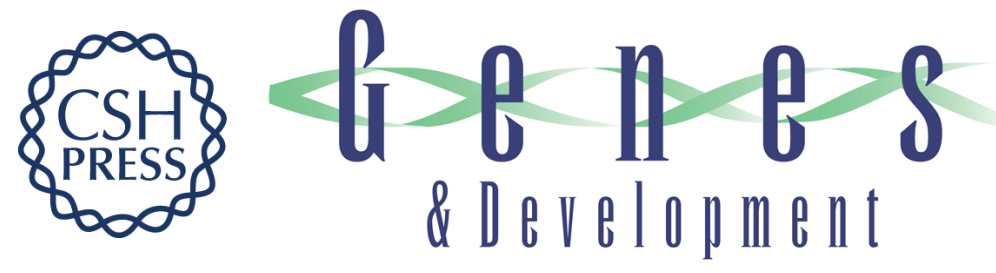

\section{Caulobacter Lon protease has a critical role in cell-cycle control of DNA methylation.}

R Wright, C Stephens, G Zweiger, et al.

Genes Dev. 1996, 10:

Access the most recent version at doi:10.1101/gad.10.12.1532

References This article cites 45 articles, 23 of which can be accessed free at:

http://genesdev.cshlp.org/content/10/12/1532.full.html\#ref-list-1

License

Email Alerting

Service

Receive free email alerts when new articles cite this article - sign up in the box at the top right corner of the article or click here.

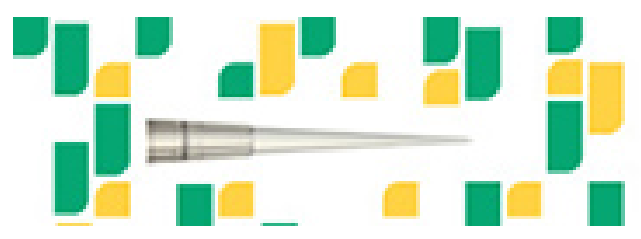

Focused on your science.

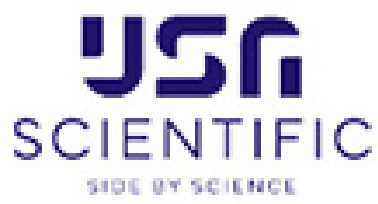

Copyright (c) Cold Spring Harbor Laboratory Press 